\title{
Existence of Positive Solutions to Nonlinear Fractional Boundary Value Problem with Changing Sign Nonlinearity and Advanced Arguments
}

\author{
Zhaocai Hao and Yubo Huang \\ School of Mathematical Sciences, Qufu Normal University, Qufu, Shandong 273165, China \\ Correspondence should be addressed to Zhaocai Hao; zchjal@163.com
}

Received 24 March 2014; Accepted 19 June 2014; Published 9 July 2014

Academic Editor: Xinguang Zhang

Copyright (c) 2014 Z. Hao and Y. Huang. This is an open access article distributed under the Creative Commons Attribution License, which permits unrestricted use, distribution, and reproduction in any medium, provided the original work is properly cited.

We discuss the existence of positive solutions to a class of fractional boundary value problem with changing sign nonlinearity and advanced arguments $D^{\alpha} x(t)+\mu h(t) f(x(a(t)))=0, t \in(0,1), 2<\alpha \leq 3, \mu>0, x(0)=x^{\prime}(0)=0, x(1)=\beta x(\eta)+\lambda[x], \beta>$ 0 , and $\eta \in(0,1)$, where $D^{\alpha}$ is the standard Riemann-Liouville derivative, $f:[0, \infty) \rightarrow[0, \infty)$ is continuous, $f(0)>0, h$ : $[0,1] \rightarrow(-\infty,+\infty)$, and $a(t)$ is the advanced argument. Our analysis relies on a nonlinear alternative of Leray-Schauder type. An example is given to illustrate our results.

\section{Introduction}

Fractional differential equations (FDEs) have been of great interest for the past three decades. It is caused both by the intensive development of the theory of fractional calculus itself and by the applications of such constructions in the modeling of many phenomena in various fields of science and engineering. Indeed, we can find numerous applications in viscoelasticity, electrochemistry, control, porous media, and so forth (see $[1,2])$. Therefore, the theory of FDEs has been developed very quickly. There has been a significant development in fractional differential equations in recent years; see [1-30].

In [5], the author studied existence of positive solutions in case of the nonlinear fractional differential equation as follows:

$$
\begin{gathered}
D^{s} u=\lambda a(t) f(u), \quad 0<t<1, \\
u(0)=0,
\end{gathered}
$$

where $0<s<1, D^{s}$ is the standard Riemann-Liouville fractional derivative, $f:[0, \infty) \rightarrow[0, \infty)$ is continuous, and $a:[0,1] \rightarrow R$. In [10], the author applied the AveryPeterson fixed point theorem to obtain sufficient conditions of the existence of multiple solutions to the following problem:

$$
\begin{aligned}
x^{\prime \prime \prime}(t)+h(t) f(x(\alpha(t)))=0, & t \in(0,1), \\
x(0)=x^{\prime \prime}(0) & =0, \\
x(1)=\beta x(\eta)+\lambda[x], \quad \beta>0, & \eta \in(0,1),
\end{aligned}
$$

where $f:[0, \infty) \rightarrow[0, \infty)$ is continuous and $h(t)$ is a nonnegative continuous function defined on $[0,1]$.

Motivated by $[5,10]$, in this paper, we consider the existence of positive solution of the following boundary value problem for nonlinear fractional differential equation with changing sign nonlinearity and advanced arguments:

$$
\begin{gathered}
D^{\alpha} x(t)+\mu h(t) f(x(a(t)))=0, \\
t \in(0,1), \quad 2<\alpha \leq 3, \quad \mu>0, \\
x(0)=x^{\prime}(0)=0, \\
x(1)=\beta x(\eta)+\lambda[x], \quad \beta>0, \eta \in(0,1),
\end{gathered}
$$

where $\lambda$ denotes a linear functional on $C[0,1]$ given by $\lambda[x]=$ $\int_{0}^{1} x(t) d \Lambda(t)$ involving a Stieltjes integral with a suitable 
function $\Lambda$ of bounded variation. It is important to indicate that we did not assume that $\lambda[x]$ is positive to all positive $x$. The measure $d \Lambda$ can be a signed measure.

Put $J=[0,1]$; let us introduce the following assumptions:

$\left(H_{1}\right) f:[0, \infty) \rightarrow[0, \infty)$ is continuous, and $f(0)>0$;

$\left(H_{2}\right) a \in C(J, J)$, and $t \leq a(t)$ on $J$;

$\left(H_{3}\right) h:[0,1] \rightarrow(-\infty,+\infty)$ may change sign; $h$ is not identically zero on any subinterval on $J$;

$\left(H_{4}\right) 0<\beta \eta^{\alpha-1}+\lambda[p]<1$, where $p(t)=t^{\alpha-1}$.

\section{Basic Definitions and Preliminaries}

In this section, we present some preliminaries and lemmas that are useful to the proof of our main results. For convenience, we also present the necessary definitions from fractional calculus theory here. These definitions can be found in the recent literature.

Definition 1. The fractional integral of order $\alpha>0$ of a function $x:(0,+\infty) \rightarrow R$ is given by

$$
I_{0+}^{\alpha} x(t)=\frac{1}{\Gamma(\alpha)} \int_{0}^{t}(t-s)^{\alpha-1} x(t) d s,
$$

provided that the right-hand side is pointwise defined on $(0,+\infty)$.

Definition 2. The fractional derivative of order $\alpha>0$ of a continuous function $x:(0,+\infty) \rightarrow R$ is given by

$$
D_{0+}^{\alpha} x(t)=\frac{1}{\Gamma(n-\alpha)}\left(\frac{d}{d t}\right)^{n} \int_{0}^{t}(t-s)^{n-\alpha-1} x(t) d s,
$$

where $n=[\alpha]+1$ and $[\alpha]$ denotes the integral part of number $\alpha$, provided that the right-hand side is pointwise defined on $(0,+\infty)$.

Lemma 3. Let $\alpha>0, x \in C(0,1) \cap L(0,1)$; then

$$
I_{0+}^{\alpha} D_{0+}^{\alpha} x(t)=x(t)+c_{1} t^{\alpha-1}+c_{2} t^{\alpha-2}+\cdots+c_{n} t^{\alpha-n},
$$

where $c_{i} \in R(i=1,2, \ldots, n), n$ being the smallest integer greater than or equal to $\alpha$.

Consider the following boundary value problem:

$$
\begin{aligned}
& D^{\alpha} x(t)+y(t)=0, \quad t \in(0,1), 2<\alpha \leq 3, \\
& x(0)=x^{\prime}(0)=0, \\
& x(1)=\beta x(\eta)+\lambda[x], \quad \beta>0, \eta \in(0,1) .
\end{aligned}
$$

Lemma 4. Assume that $\beta \eta^{\alpha-1} \neq 1$ and $y \in C(J, R)$; then problem (7) has the unique solution given by the following formula:

$$
\begin{aligned}
x(t)= & \frac{t^{\alpha-1}}{1-\beta \eta^{\alpha-1}} \lambda[x]+\frac{\beta t^{\alpha-1}}{1-\beta \eta^{\alpha-1}} \int_{0}^{1} k(\eta, s) y(s) d s \\
& +\int_{0}^{1} k(t, s) y(s) d s,
\end{aligned}
$$

where

$$
k(t, s)=\frac{1}{\Gamma(\alpha)} \begin{cases}{[t(1-s)]^{\alpha-1},} & 0 \leq t \leq s \leq 1 \\ {[t(1-s)]^{\alpha-1}-(t-s)^{\alpha-1},} & 0 \leq s \leq t \leq 1 .\end{cases}
$$

Theorem 5. Let $X$ be a Banach space with $C \subset X$ closed and convex. Assume that $U$ is a relatively open subset of $C$ with $0 \in$ $C$ and $A: \bar{U} \rightarrow C$ is a continuous, compact map. Then either

(i) A has a fixed point in $\bar{U}$ or

(ii) there exist $u \in \partial U$ and $\tau \in(0,1)$ with $u=\tau A u$.

\section{Existence of Positive Solutions}

Let us denote by $X=C[0,1]$ the Banach space of all continuous real functions on $[0,1]$ endowed with the sup norm and let $K$ be the cone:

$$
K=\{x \in X, x(t) \geq 0, t \in J\} .
$$

Lemma 6. Let assumptions $\left(H_{1}\right)-\left(H_{4}\right)$ hold. Moreover, we assume that assumptions $\left(\mathrm{H}_{5}\right)-\left(\mathrm{H}_{6}\right)$ hold with

$$
\begin{aligned}
& \left(H_{5}\right) \int_{0}^{1} d \Lambda(t) \geq 0, \int_{0}^{1} t^{\alpha-1} d \Lambda(t) \geq 0 \text { and } \kappa(s)= \\
& \int_{0}^{1} k(t, s) d \Lambda(t) \geq 0, \\
& \left(H_{6}\right) \begin{array}{l}
h:[0,1] \rightarrow(-\infty,+\infty) \text { is continuous, } h(0) \neq 0, \text { and } \\
\text { there is } \sigma>1 \text { such that }
\end{array} \\
& \frac{t^{\alpha-1}}{\Delta-\rho}\left(\frac{\beta \rho}{\Delta} \int_{0}^{1} k(\eta, s) h^{+}(s) d s+\int_{0}^{1} \kappa(s) h^{+}(s) d s\right) \\
& +\int_{0}^{1} k(t, s) h^{+}(s) d s+\frac{\beta t^{\alpha-1}}{\Delta} \int_{0}^{1} k(\eta, s) h^{+}(s) d s \\
& \geq \sigma \frac{t^{\alpha-1}}{\Delta-\rho}\left(\frac{\beta \rho}{\Delta} \int_{0}^{1} k(\eta, s) h^{-}(s) d s+\int_{0}^{1} \kappa(s) h^{-}(s) d s\right) \\
& \left.\quad+\int_{0}^{1} k(t, s) h^{-}(s) d s+\frac{\beta t^{\alpha-1}}{\Delta} \int_{0}^{1} k(\eta, s) h^{-}(s) d s\right],
\end{aligned}
$$

where $\Delta=1-\beta \eta^{\alpha-1}, \rho=\lambda[p], h^{+}(t)=\max \{0$, $h(t)\}$ and $h^{-}(t)=\max \{0,-h(t)\}$. Then, for every $0<$ $\delta<1$, there exists a positive number $\bar{\mu}$ such that, for $0<\mu<\bar{\mu}$, the nonlinear fractional differential equation,

$$
\begin{gathered}
D^{\alpha} x(t)+\mu h^{+}(t) f(x(a(t)))=0, \\
t \in(0,1), \quad 2<\alpha \leq 3, \quad \mu>0, \\
x(0)=x^{\prime}(0)=0, \\
x(1)=\beta x(\eta)+\lambda[x], \quad \beta>0, \quad \eta \in(0,1),
\end{gathered}
$$

has a positive solution $\bar{x}_{\mu}$ with $\left\|\bar{x}_{\mu}\right\| \rightarrow 0$ as $\mu \rightarrow 0$ and

$$
\bar{x}_{\mu}(t) \geq \mu \delta f(0) m(t),
$$


where

$$
\begin{aligned}
m(t)= & \frac{t^{\alpha-1}}{\Delta-\rho}\left(\frac{\beta \rho}{\Delta} \int_{0}^{1} k(\eta, s) h^{+}(s) d s+\int_{0}^{1} \kappa(s) h^{+}(s) d s\right) \\
& +\frac{\beta t^{\alpha-1}}{\Delta} \int_{0}^{1} k(\eta, s) h^{+}(s) d s+\int_{0}^{1} k(t, s) h^{+}(s) d s .
\end{aligned}
$$

Proof. It is easy to know from $(9),\left(H_{5}\right)$, and $\left(H_{6}\right)$ that $m(t)>$ $0, t \in(0,1]$. By Lemma $4,(12)$ has a unique solution in $X$ :

$$
\begin{aligned}
x(t)= & \frac{t^{\alpha-1}}{1-\beta \eta^{\alpha-1}} \lambda[x] \\
& +\frac{\beta t^{\alpha-1}}{1-\beta \eta^{\alpha-1}} \mu \int_{0}^{1} k(\eta, s) h^{+}(s) f(x(a(s))) d s \\
& +\mu \int_{0}^{1} k(t, s) h^{+}(s) f(x(a(s))) d s .
\end{aligned}
$$

For $x \in C\left(J, R_{+}\right)$, we define two operators $T$ and $S$ by

$$
\begin{aligned}
& T x(t)=\frac{t^{\alpha-1}}{\Delta} \lambda[x]+\mu F x(t), \\
& S x(t)=\frac{t^{\alpha-1}}{\Delta-\rho} \mu \lambda[F x]+\mu F x(t),
\end{aligned}
$$

where

$$
\begin{aligned}
F x(t)= & \frac{\beta t^{\alpha-1}}{\Delta} \int_{0}^{1} k(\eta, s) h^{+}(s) f(x(a(s))) d s \\
& +\int_{0}^{1} k(t, s) h^{+}(s) f(x(a(s))) d s, \\
\lambda[F x]= & \frac{\beta \rho}{\Delta} \int_{0}^{1} k(\eta, s) h^{+}(s) f(x(a(s))) d s \\
& +\int_{0}^{1} \kappa(s) h^{+}(s) f(x(a(s))) d s .
\end{aligned}
$$

It is easy to show that $T: K \rightarrow K$ and $S: K \rightarrow K$ are completely continuous. We claim that operators $T$ and $S$ have the same fixed points in $K$. In fact, let $x=S x$; then

$$
\lambda[x]=\frac{\rho}{\Delta-\rho} \mu \lambda[F x]+\mu \lambda[F x]=\frac{\Delta}{\Delta-\rho} \mu \lambda[F x] .
$$

So

$$
\begin{aligned}
x(t) & =S x(t)=\frac{t^{\alpha-1}}{\Delta-\rho} \mu \lambda[F x]+\mu F x(t) \\
& =\frac{t^{\alpha-1}}{\Delta} \lambda[x]+\mu F x(t)=T x(t) .
\end{aligned}
$$

Let $x=T x$; then $\lambda[x]=(\rho / \Delta) \lambda[x]+\mu \lambda[F x]$. So $\lambda[x]=$ $(\Delta /(\Delta-\rho)) \mu \lambda[F x]$, and hence

$$
\begin{aligned}
x(t) & =T x(t)=\frac{t^{\alpha-1}}{\Delta} \lambda[x]+\mu F x(t) \\
& =\frac{t^{\alpha-1}}{\Delta-\rho} \mu \lambda[F x]+\mu F x(t)=S x(t) .
\end{aligned}
$$

This shows that fixed points of $S$ are solutions of (12). We will apply the nonlinear alternative of Leray-Schauder type to prove that $S$ has at least one fixed point for small $\mu$.

Let $\epsilon>0$ be such that

$$
f(x(a(t))) \geq \delta f(0), \quad 0 \leq x(a(t)) \leq \epsilon, \quad \forall t \in[0,1] .
$$

Suppose that $0<\mu<\epsilon / 2\|m\| \bar{f}(\epsilon):=\bar{\mu}$, where $\bar{f}(t)=$ $\max _{0 \leq s \leq t} f(s)$; then

$$
\bar{f}(\|x\|)=\max _{0 \leq|x(a(t))| \leq\|x\|} f(x(a(t))), \quad \forall t \in[0,1] .
$$

Since $\lim _{t \rightarrow 0^{+}}(\bar{f}(t) / t)=+\infty, \bar{f}(\epsilon) / \epsilon<1 / 2 \mu\|m\|$, there exists a unique $R_{\mu} \in(0, \epsilon)$ such that

$$
\frac{\bar{f}\left(R_{\mu}\right)}{R_{\mu}}=\frac{1}{2 \mu\|m\|} .
$$

Let $x \in K$ and $\tau \in(0,1)$ be such that $x=\tau S x$. We claim that $\|x\| \neq R_{\mu}$. In fact,

$x(t)$

$=\frac{\tau t^{\alpha-1}}{\Delta-\rho} \mu\left(\frac{\beta \rho}{\Delta} \int_{0}^{1} k(\eta, s) h^{+}(s) f(x(a(s))) d s\right.$

$$
\begin{array}{r}
\left.+\int_{0}^{1} \kappa(s) h^{+}(s) f(x(a(s))) d s\right) \\
+\tau \mu\left(\frac{\beta t^{\alpha-1}}{\Delta} \int_{0}^{1} k(\eta, s) h^{+}(s) f(x(a(s))) d s\right.
\end{array}
$$

$$
\left.+\int_{0}^{1} k(t, s) h^{+}(s) f(x(a(s))) d s\right)
$$$$
\leq \frac{t^{\alpha-1}}{\Delta-\rho} \mu \bar{f}(\|x\|)
$$$$
\times\left(\int_{0}^{1} k(\eta, s) h^{+}(s) d s+\int_{0}^{1} \kappa(s) h^{+}(s) d s\right)
$$$$
+\mu \bar{f}(\|x\|)
$$$$
\times\left(\frac{\beta t^{\alpha-1}}{\Delta} \int_{0}^{1} k(\eta, s) h^{+}(s) d s+\int_{0}^{1} k(t, s) h^{+}(s) d s\right)
$$$$
=\mu \bar{f}(\|x\|) m(t) \leq \mu \bar{f}(\|x\|)\|m\| \text {. }
$$

That is, $\bar{f}(\|x\|) /\|x\| \geq 1 / \mu\|m\|$, which implies that $\|x\| \neq R_{\mu}$. Let $U=\left\{x \in K:\|x\|<R_{\mu}\right\}$. By Theorem 5, $S$ has a fixed point $\bar{x}_{\mu} \in \bar{U}$. Moreover, combining (21) with the expression of operator $S$, we obtain that

$$
\bar{x}_{\mu}(t) \geq \mu \delta f(0) m(t), \quad \forall t \in(0,1] .
$$

Hence (12) has a positive solution $\bar{x}_{\mu}(t)$. Note that $R_{\mu} \rightarrow 0$ as $\mu \rightarrow 0$; we get that $\left\|\bar{x}_{\mu}\right\| \rightarrow 0$ as $\mu \rightarrow 0$. 
Theorem 7. Suppose that $\left(H_{1}\right)-\left(H_{6}\right)$ hold. Then there exists a positive number $\mu^{*}>0$ such that (3) has at least one positive solution for $\mu \in\left(0, \mu^{*}\right)$.

Proof. Let

$$
\begin{aligned}
\omega(t)= & \frac{t^{\alpha-1}}{\Delta-\rho}\left(\frac{\beta \rho}{\Delta} \int_{0}^{1} k(\eta, s) h^{-}(s) d s+\int_{0}^{1} \kappa(s) h^{-}(s) d s\right) \\
& +\frac{\beta t^{\alpha-1}}{\Delta} \int_{0}^{1} k(\eta, s) h^{-}(s) d s+\int_{0}^{1} k(t, s) h^{-}(s) d s
\end{aligned}
$$

Then $\omega(t) \geq 0$ for each $t \in(0,1]$. We have $m(t) \geq \sigma \omega(t), \sigma>$ 1 . Choose $c \in(0,1)$ such that $\sigma c>1$. There is $b>0$ such that $f(x(a(t))) \leq \sigma c f(0)$ for $x \in[0, b]$; then

$$
\omega(t) f(x(a(t))) \leq c m(t) f(0) \quad \text { for } t \in(0,1], x \in[0, b] .
$$

Fix $\delta \in(c, 1)$, and let $\mu^{*}>0$ be such that

$$
\left\|\bar{x}_{\mu}\right\|+\mu \delta f(0)\|m\| \leq b, \quad \mu \in\left(0, \mu^{*}\right)
$$

where $\bar{x}_{\mu}$ is given by Lemma 6 , and

$$
\left|f\left(x_{1}(a(t))\right)-f\left(x_{2}(a(t))\right)\right| \leq f(0) \frac{\delta-c}{2},
$$

for $x_{1}, x_{2} \in[0, b]$ with $\left|x_{1}-x_{2}\right| \leq \mu^{*} \delta f(0)\|m\|$.

Let $\mu \in\left(0, \mu^{*}\right)$. We look for a solution $x_{\mu}$ of the form $\bar{x}_{\mu}+v_{\mu}$, where $\bar{x}_{\mu}$ is the solution of (12), given by Lemma 6 . Thus $v_{\mu}$ solves the following equation:

$$
\begin{gathered}
D^{\alpha} v_{\mu}=\mu h^{+}(t)\left(f_{1}^{\prime}-f_{2}^{\prime}\right)-\mu h^{-}(t) f_{1}^{\prime} \\
v_{\mu}(0)=v_{\mu}^{\prime}(0)=0 \\
v_{\mu}(1)=\beta v_{\mu}(\eta)+\lambda\left[v_{\mu}\right]
\end{gathered}
$$

where $f_{1}^{\prime}=f\left(\bar{x}_{\mu}(a(t))+v_{\mu}(a(t))\right), f_{2}^{\prime}=f\left(\bar{x}_{\mu}(a(t))\right)$.

Now, we need to prove the existence of $v_{\mu}$. Consider the following equation:

$$
\begin{gathered}
D^{\alpha} v=\mu h^{+}(t)\left(f_{1}-f_{2}\right)-\mu h^{-}(t) f_{1}, \\
v(0)=v^{\prime}(0)=0, \\
v(1)=\beta v(\eta)+\lambda[v],
\end{gathered}
$$

where

$$
f_{1}=f\left(\bar{x}_{\mu}(a(t))+v(a(t))\right), \quad f_{2}=f\left(\bar{x}_{\mu}(a(t))\right) .
$$

Obviously, (31) is equivalent to the operator equation:

$S v(t)$

$$
\begin{aligned}
& =\frac{t^{\alpha-1}}{\Delta-\rho} \mu\left(\frac{\beta \rho}{\Delta} \int_{0}^{1} k(\eta, s) h^{+}(s)\left(f_{1}-f_{2}\right) d s\right. \\
& \left.+\int_{0}^{1} \kappa(s) h^{+}(s)\left(f_{1}-f_{2}\right) d s\right) \\
& +\mu\left(\frac{\beta t^{\alpha-1}}{\Delta} \int_{0}^{1} k(\eta, s) h^{+}(s)\left(f_{1}-f_{2}\right) d s\right. \\
& \left.+\int_{0}^{1} k(t, s) h^{+}(s)\left(f_{1}-f_{2}\right) d s\right) \\
& -\frac{t^{\alpha-1}}{\Delta-\rho} \mu\left(\frac{\beta \rho}{\Delta} \int_{0}^{1} k(\eta, s) h^{-}(s) f_{1} d s\right. \\
& \left.+\int_{0}^{1} \kappa(s) h^{-}(s) f_{1} d s\right) \\
& +\mu\left(\frac{\beta t^{\alpha-1}}{\Delta} \int_{0}^{1} k(\eta, s) h^{-}(s) f_{1} d s\right. \\
& \left.+\int_{0}^{1} k(t, s) h^{-}(s) f_{1} d s\right) .
\end{aligned}
$$

It is easy to show that operator $S: X \rightarrow X$ is completely continuous. Let $v \in X$ and $\tau \in(0,1)$ such that $v=\tau S v$. That is,

$$
\begin{array}{r}
v(t) \\
=\frac{\tau t^{\alpha-1}}{\Delta-\rho} \mu\left(\frac{\beta \rho}{\Delta} \int_{0}^{1} k(\eta, s) h^{+}(s)\left(f_{1}-f_{2}\right) d s\right. \\
\left.+\int_{0}^{1} \kappa(s) h^{+}(s)\left(f_{1}-f_{2}\right) d s\right) \\
+\tau \mu\left(\frac{\beta t^{\alpha-1}}{\Delta} \int_{0}^{1} k(\eta, s) h^{+}(s)\left(f_{1}-f_{2}\right) d s\right. \\
\left.+\int_{0}^{1} k(t, s) h^{+}(s)\left(f_{1}-f_{2}\right) d s\right) \\
-\frac{\tau t^{\alpha-1}}{\Delta-\rho} \mu\left(\frac{\beta \rho}{\Delta} \int_{0}^{1} k(\eta, s) h^{-}(s) f_{1} d s\right. \\
\left.+\int_{0}^{1} \kappa(s) h^{-}(s) f_{1} d s\right) \\
+\tau \mu\left(\frac{\beta t^{\alpha-1}}{\Delta} \int_{0}^{1} k(\eta, s) h^{-}(s) f_{1} d s\right. \\
\left.k(t, s) h^{-}(s) f_{1} d s\right) .
\end{array}
$$


We claim that $\|v\| \neq \mu \delta f(0)\|m\|$. Suppose on the contrary that $\|v\|=\mu \delta f(0)\|m\|$. Then, by (28) and (29), we get

$$
\begin{gathered}
\left\|\bar{x}_{\mu}+v\right\| \leq\left\|\bar{x}_{\mu}\right\|+\|v\| \leq b, \\
\left|f_{1}-f_{2}\right| \leq f(0) \frac{\delta-c}{2} .
\end{gathered}
$$

From (27), we get

$$
\omega(t) f(x(a(t))) \leq c m(t) f(0), \quad t \in(0,1] .
$$

Using (34)-(36), for each $t \in(0,1]$, we obtain that

$|v(t)|$

$$
\begin{aligned}
& \leq \frac{t^{\alpha-1}}{\Delta-\rho} \mu\left(\frac{\beta \rho}{\Delta} \int_{0}^{1} k(\eta, s) h^{+}(s)\left|f_{1}-f_{2}\right| d s\right. \\
& \left.+\int_{0}^{1} \kappa(s) h^{+}(s)\left|f_{1}-f_{2}\right| d s\right) \\
& +\mu\left(\frac{\beta t^{\alpha-1}}{\Delta} \int_{0}^{1} k(\eta, s) h^{+}(s)\left|f_{1}-f_{2}\right| d s\right. \\
& +\frac{t^{\alpha-1}}{\Delta-\rho} \mu\left(\frac{\beta \rho}{\Delta} \int_{0}^{1} k(\eta, s) h^{-}(s) f_{1} d s\right. \\
& +\frac{t^{\alpha-1}}{\Delta-\rho)} \mu \bar{f}(b)\left(\frac{\beta \rho}{\Delta} \int_{0}^{1} k(\eta, s) h^{-}(s) d s\right. \\
& \left.+\frac{(\beta \rho}{\Delta} \int_{0}^{1} k(\eta, s) h^{+}(s) d s+f_{0}^{1} \kappa(s) h^{+}(s) d s\right) \\
& +\mu\left(\frac{\beta t^{\alpha-1}}{\Delta} \int_{0}^{1} k(\eta, s) h^{-}(s) f_{1} d s\right. \\
& \left.+\int_{0}^{1} k(t, s) h^{-}(s) f_{1} d s\right) \\
& \left.+\mu f(0) \frac{\delta-c}{2}(t, s) h^{+}(s) d s\right)
\end{aligned}
$$

$$
\begin{aligned}
& \quad+\mu \bar{f}(b)\left(\frac{\beta t^{\alpha-1}}{\Delta} \int_{0}^{1} k(\eta, s) h^{-}(s) d s\right. \\
& \left.\quad+\int_{0}^{1} k(t, s) h^{-}(s) d s\right) \\
& =\mu f(0) \frac{\delta-c}{2} m(t)+\mu \bar{f}(b) \omega(t) \\
& \leq \mu f(0) \frac{\delta-c}{2} m(t)+\mu c f(0) m(t) \\
& =\mu f(0) \frac{\delta+c}{2} m(t) .
\end{aligned}
$$

In particular,

$$
\|v\| \leq \mu f(0) \frac{\delta+c}{2}\|m\|<\mu f(0) \delta\|m\|,
$$

which is a contradiction. And so the claim is proved. Let $U=$ $\{x \in X:\|x\|<\mu \delta f(0)\|m\|\}$. By Theorem $5, S$ has a fixed point $v_{\mu} \in \bar{U}$. Consequently, $\left\|v_{\mu}\right\| \leq \mu \delta f(0)\|m\|$. This proves that there exists $v_{\mu}$ this is the solution of (30). Hence $v_{\mu}$ satisfies (37) and Lemma 6; then we get

$$
\begin{aligned}
x_{\mu}(t) & \geq \bar{x}_{\mu}(t)-\left|v_{\mu}(t)\right| \geq \mu \delta f(0) m(t)-\mu f(0) \frac{\delta+c}{2} m(t) \\
& =\mu f(0) \frac{\delta-c}{2} m(t)>0 ;
\end{aligned}
$$

that is, $x_{\mu}$ is a positive solution of (3). So the proof of Theorem 7 is complete.

\section{An Example}

In this section, we give an example to illustrate the result of this paper. Consider the following nonlinear fractional differential equation:

$$
\begin{gathered}
D^{5 / 2} x(t)-\mu\left(\frac{4}{5}-t\right)\left(x^{(4)}(\sqrt{t})+\sin ^{2} x(\sqrt{t})+\frac{1}{10}\right)=0, \\
x(0)=x^{\prime}(0)=0 \\
x(1)=\frac{1}{2} x\left(\frac{1}{2}\right)+\int_{0}^{1} x(t)(3 t-1) d t .
\end{gathered}
$$

Let $f(x(a(t)))=x^{(4)}(\sqrt{t})+\sin ^{2} x(\sqrt{t})+1 / 10, a(t)=\sqrt{t}$ and $h(t)=4 / 5-t$. Obviously, all assumptions $\left(H_{1}\right)-\left(H_{3}\right)$ hold. In the following, we will verify that assumptions $\left(H_{4}\right)-\left(H_{6}\right)$ hold also.

(i) It is obvious that

$$
\begin{aligned}
\beta \eta^{\alpha-1}+\lambda[p]= & \beta \eta^{\alpha-1}+\int_{0}^{1} t^{\alpha-1}(3 t-1) d t=\beta \eta^{\alpha-1} \\
& +\frac{2 \alpha-1}{\alpha(\alpha+1)}=\frac{1}{4 \sqrt{2}}+\frac{16}{35}
\end{aligned}
$$

implies $\left(H_{4}\right)$. 
(ii) By direct calculation, we have

$$
\begin{aligned}
\int_{0}^{1}(3 t-1) d t=\frac{1}{2}, \quad \int_{0}^{1} t^{\alpha-1}(3 t-1) d t=\frac{16}{35} \\
\kappa(s)=\int_{0}^{1} k(t, s)(3 t-1) d t \\
=\frac{1}{\alpha(\alpha+1)}(1-s)^{\alpha-1} s(3 s+2 \alpha-4) \\
=\frac{4}{35}(1-s)^{3 / 2} s(3 s+1) \geq 0
\end{aligned}
$$

so assumption $\left(\mathrm{H}_{5}\right)$ holds.

(iii) Finally, we check assumption $\left(H_{6}\right)$. It means that there exists $\epsilon>0$ such that $m(t) \geq(1+\epsilon) \omega(t), t \in(0,1]$. Note that

$$
\begin{gathered}
h^{+}(t)=\max \{0, h(t)\}= \begin{cases}\frac{4}{5}-t, & 0 \leq t \leq \frac{4}{5}, \\
0, & \frac{4}{5}<t \leq 1,\end{cases} \\
h^{-}(t)=\max \{0,-h(t)\}= \begin{cases}0, & 0 \leq t \leq \frac{4}{5}, \\
t-\frac{4}{5}, & \frac{4}{5}<t \leq 1 .\end{cases}
\end{gathered}
$$

We now verify that there exists $\epsilon_{1}>0$ such that

$$
\begin{aligned}
& \frac{t^{\alpha-1}}{\Delta-\rho} \frac{\beta \rho}{\Delta} \int_{0}^{1} k(\eta, s) h^{+}(s) d s \\
& \quad \geq\left(1+\epsilon_{1}\right) \frac{t^{\alpha-1}}{\Delta-\rho} \frac{\beta \rho}{\Delta} \int_{0}^{1} k(\eta, s) h^{-}(s) d s, \quad t \in(0,1]
\end{aligned}
$$

that is,

$$
\begin{array}{r}
\int_{0}^{1} k\left(\frac{1}{2}, s\right)\left(\frac{4}{5}-s\right) d s \geq \epsilon_{1} \int_{4 / 5}^{1} k\left(\frac{1}{2}, s\right)\left(s-\frac{4}{5}\right) d s \\
t \in(0,1]
\end{array}
$$

By simple calculation, we get

$$
\begin{gathered}
\int_{0}^{1} k\left(\frac{1}{2}, s\right)\left(\frac{4}{5}-s\right) d s=\frac{13 \sqrt{2}}{700} \\
\int_{4 / 5}^{1} k\left(\frac{1}{2}, s\right)\left(s-\frac{4}{5}\right) d s=\frac{2}{7 \times 5^{4} \times \sqrt{10}} .
\end{gathered}
$$

Setting $\epsilon_{1} \in\left(0,\left(13 \times 5^{2} \times \sqrt{5}\right) / 4\right)$, then inequality (44) holds. Similarly, there exists $\epsilon_{2}, \epsilon_{3}, \epsilon_{4}>0$ such that

$$
\begin{aligned}
& \frac{t^{\alpha-1}}{\Delta-\rho} \int_{0}^{1} \kappa(s) h^{+}(s) d s \\
& \quad \geq\left(1+\epsilon_{2}\right) \frac{t^{\alpha-1}}{\Delta-\rho} \int_{0}^{1} \kappa(s) h^{-}(s) d s, \quad t \in(0,1], \\
& \frac{\beta t^{\alpha-1}}{\Delta} \int_{0}^{1} k(\eta, s) h^{+}(s) d s \\
& \quad \geq\left(1+\epsilon_{3}\right) \frac{\beta t^{\alpha-1}}{\Delta} \int_{0}^{1} k(\eta, s) h^{-}(s) d s, \quad t \in(0,1],
\end{aligned}
$$

$$
\begin{aligned}
& \int_{0}^{1} k(t, s) h^{+}(s) d s \\
& \quad \geq\left(1+\epsilon_{4}\right) \int_{0}^{1} k(t, s) h^{-}(s) d s, \quad t \in(0,1] .
\end{aligned}
$$

Let $\epsilon=\min \left\{\epsilon_{1}, \epsilon_{2}, \epsilon_{3}, \epsilon_{4}\right\}$. By (44)-(47), we obtain that there exists $\epsilon>0$ such that

$$
m(t) \geq(1+\epsilon) \omega(t), \quad t \in(0,1] .
$$

Thus assumption $\left(\mathrm{H}_{6}\right)$ holds. By applying Theorem 7 , we know that there exists a number $\mu^{*}>0$ such that (40) has at least one positive solution for $\mu \in\left(0, \mu^{*}\right)$.

\section{Conflict of Interests}

The authors declare that there is no conflict of interests regarding the publication of this paper.

\section{Acknowledgments}

Zhaocai Hao acknowledges the support from NSFC (11371221) and the Education Department of Shandong Province Science and Technology Plan Project (J13LI01). The authors are grateful to the anonymous referees for their helpful suggestions and comments.

\section{References}

[1] R. Hilfer, Applications of Fractional Calculus in Physics, World Scientific, 2000.

[2] F. Metzler, W. Schick, H. G. Kilian, and T. F. Nonnenmacher, "Relaxation in filled polymers: a fractional calculus approach," The Journal of Chemical Physics, vol. 103, no. 16, pp. 7180-7186, 1995.

[3] R. P. Agarwal, M. Benchohra, and B. Slimani, "Existence results for differential equations with fractional order and impulses," Georgian Academy of Sciences A: Razmadze Mathematical Institute: Memoirs on Differential Equations and Mathematical Physics, vol. 44, pp. 1-21, 2008.

[4] E. Ahmed and H. A. El-Saka, "On fractional order models for Hepatitis C," Nonlinear Biomedical Physics, vol. 4, article 1, 2010.

[5] C. Bai, "Positive solutions for nonlinear fractional differential equations with coefficient that changes sign," Nonlinear Analysis: Theory, Methods and Applications, vol. 64, no. 4, pp. 677-685, 2006. 
[6] Z. Bai, "On positive solutions of a nonlocal fractional boundary value problem," Nonlinear Analysis: Theory, Methods \& Applications, vol. 72, no. 2, pp. 916-924, 2010.

[7] K. Diethelm and A. D. Freed, "On the solution of nonlinear fractional order differential equations used in the modeling of viscoplasticity," in Scientific Computing in Chemical Engineering II-Computational Fluid Dynamics, Reaction Engineering and Molecular Properties, F. Keil, W. Mackens, H. Voss, and J. Werther, Eds., pp. 217-307, Springer, Heidelberg, Germany, 1999.

[8] X. Ding and Y. Jiang, "Waveform relaxation methods for fractional functional differential equations," Fractional Calculus and Applied Analysis, vol. 16, no. 3, pp. 573-594, 2013.

[9] L. Gaul, P. Klein, and S. Kemple, "Damping description involving fractional operators," Mechanical Systems and Signal Processing, vol. 5, no. 2, pp. 81-88, 1991.

[10] T. Jankowski, "Existence of positive solutions to third order differential equations with advanced arguments and nonlocal boundary conditions," Nonlinear Analysis, vol. 75, no. 2, pp. 913923, 2012.

[11] T. Jankowski, "Positive solutions for second order impulsive differential equations involving Stieltjes integral conditions," Nonlinear Analysis: Theory, Methods and Applications, vol. 74, no. 11, pp. 3775-3785, 2011.

[12] A. A. Kilbas, H. M. Srivastava, and J. J. Trujillo, Theory and Applications of Fractional Differential Equations, vol. 204 of North-Holland Mathematics Studies, Elsevier Science, Amsterdam, The Netherlands, 2006.

[13] A. A. Kilbas and J. J. Trujillo, "Differential equations of fractional order: methods, results and problems. I," Applicable Analysis, vol. 78, no. 1-2, pp. 153-192, 2001.

[14] A. A. Kilbas and J. J. Trujillo, "Differential equations of fractional order: methods, results and problems. II," Applicable Analysis, vol. 81, no. 2, pp. 435-493, 2002.

[15] R. C. Koeller, "Applications of fractional calculus to the theory of viscoelasticity," Journal of Applied Mechanics, vol. 51, no. 2, pp. 299-307, 1984.

[16] C. Kou, H. Zhou, and Y. Yan, "Existence of solutions of initial value problems for nonlinear fractional differential equations on the half-axis," Nonlinear Analysis, vol. 74, no. 17, pp. 59755986, 2011.

[17] P. Kumar and O. P. Agrawal, "An approximate method for numerical solution of fractional differential equations," Signal Processing, vol. 86, no. 10, pp. 2602-2610, 2006.

[18] V. Lakshmikantham, S. Leela, and J. Vasundhara, Theory of Fractional Dynamic Systems, Cambridge Academic, Cambridge, UK, 2009.

[19] V. Lakshmikantham and A. S. Vatsala, "Basic theory of fractional differential equations," Nonlinear Analysis: Theory, Methods \& Applications, vol. 69, no. 8, pp. 2677-2682, 2008.

[20] K. S. Miller and B. Ross, An Introduction to the Fractional Calculus and Differential Equations, John Wiley and Sons, New York, NY, USA, 1993.

[21] I. Podlubny, Fractional Differential Equations: An Introduction to Fractional Derivatives, Fractional Differential Equations, to Methods of their Solution and Some of their Applications, Academic Press, San Diego, Calif, USA, 1999.

[22] I. Podlubny, "Geometric and physical interpretation of fractional integration and fractional differentiation," Fractional Calculus and Applied Analysis, vol. 5, no. 4, pp. 367-386, 2002.
[23] H. M. Srivastava and R. K. Saxena, "Operators of fractional integration and their applications," Applied Mathematics and Computation, vol. 118, no. 1, pp. 1-52, 2001.

[24] X. Zhang, L. Liu, and Y. Wu, "Multiple positive solutions of a singular fractional differential equation with negatively perturbed term," Mathematical and Computer Modelling, vol. 55, no. 3-4, pp. 1263-1274, 2012.

[25] X. Zhang, L. Liu, and Y. Wu, "Existence results for multiple positive solutions of nonlinear higher order perturbed fractional differential equations with derivatives," Applied Mathematics and Computation, vol. 219, no. 4, pp. 1420-1433, 2012.

[26] Y. Wang, L. Liu, and Y. Wu, "Positive solutions for a class of fractional boundary value problem with changing sign nonlinearity," Nonlinear Analysis: Theory, Methods \& Applications, vol. 74, no. 17, pp. 6434-6441, 2011.

[27] X. Zhang, L. Liu, and Y. Wu, "The eigenvalue problem for a singular higher order fractional differential equation involving fractional derivatives," Applied Mathematics and Computation, vol. 218, no. 17, pp. 8526-8536, 2012.

[28] X. Zhang, L. Liu, and Y. Wu, "The uniqueness of positive solution for a singular fractional differential system involving derivatives," Communications in Nonlinear Science and Numerical Simulation, vol. 18, no. 6, pp. 1400-1409, 2013.

[29] X. Zhang, L. Liu, B. Wiwatanapataphee, and Y. Wu, "Positive solutions of eigenvalue problems for a class of fractional differential equations with derivatives," Abstract and Applied Analysis, vol. 2012, Article ID 512127, 16 pages, 2012.

[30] X. Zhang, L. Liu, Y. Wu, and Y. Lu, “The iterative solutions of nonlinear fractional differential equations," Applied Mathematics and Computation, vol. 219, no. 9, pp. 4680-4691, 2013. 


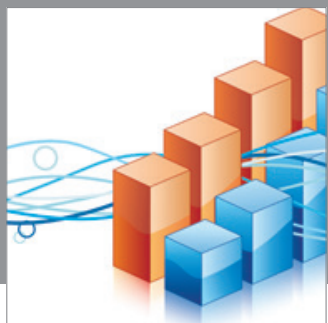

Advances in

Operations Research

mansans

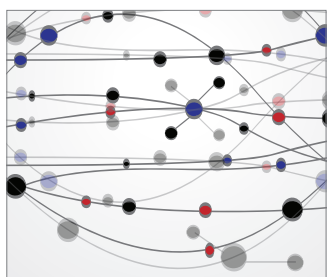

The Scientific World Journal
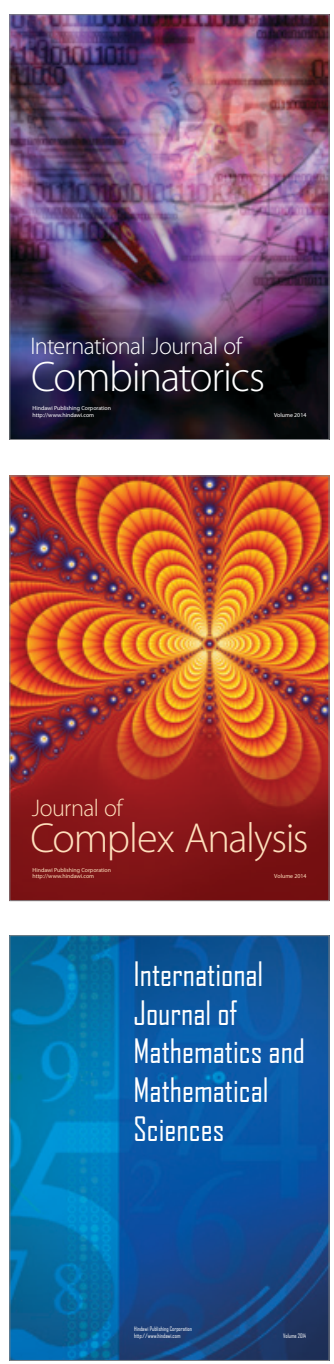
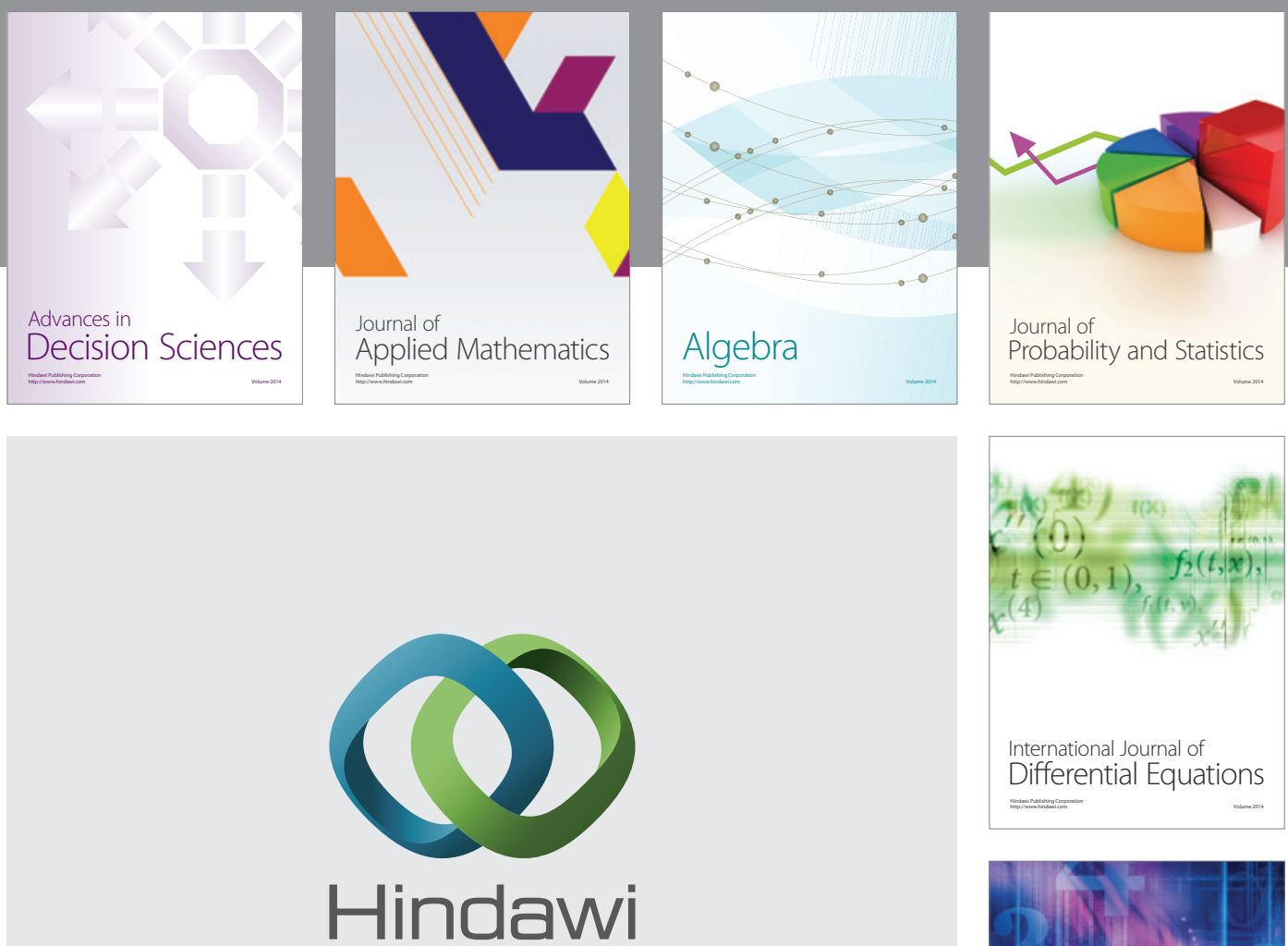

Submit your manuscripts at http://www.hindawi.com
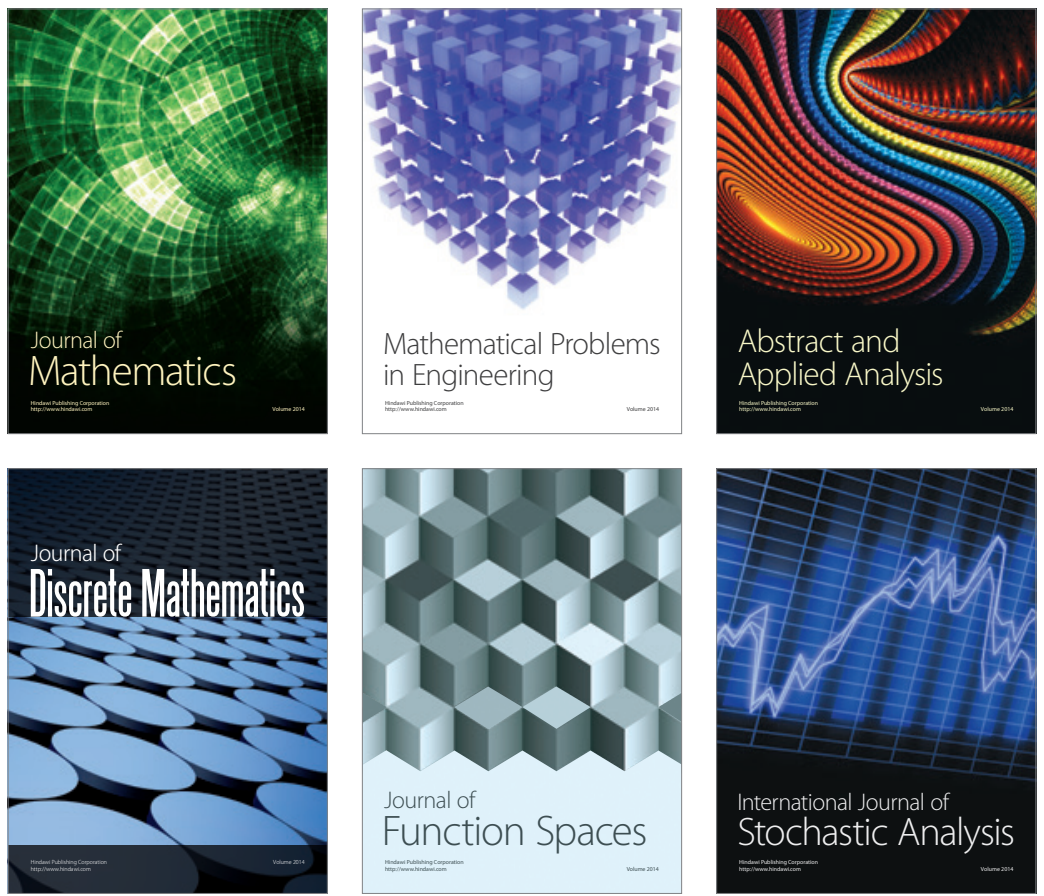

Journal of

Function Spaces

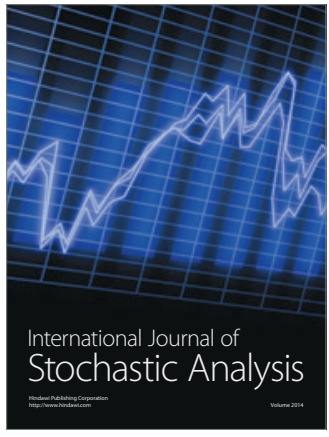

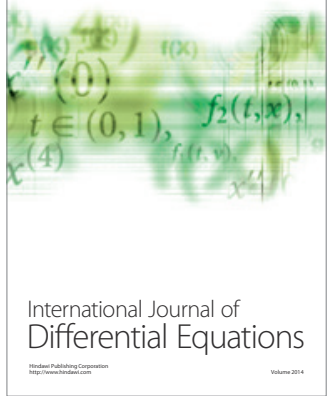
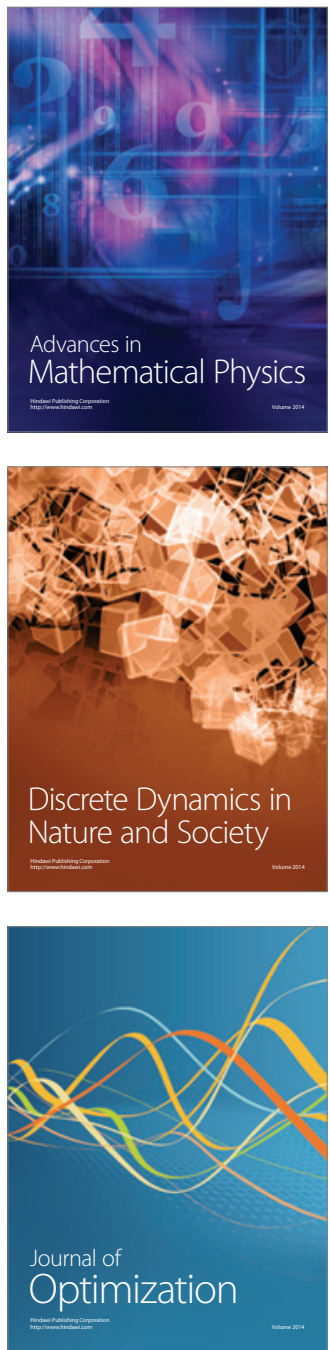